\title{
PROBLEMÁTICA, METODOLOGÍA Y TEORÍA: EL RECORRIDO DE UN GRUPO DE INVESTIGACIÓN EN ENSENANZA DE LAS CIENCIAS
}

\author{
DIBAR URE, M.C. \\ Instituto de Física. Universidad Federal Fluminense. Brasil
}

\section{SUMMARY}

This paper discusses some results obtained from research on the teaching of mechanics to initial university courses in Brazil, together with the epistemological problems arising.

Discutiremos, en este trabajo, ciertos puntos de la evolución de una línea de investigación sobre enseñanza de Mecánica Básica a jóvenes universitarios brasileños. Las dificultades encontradas no son características solamente del Brasil, sino que enfrentan a muchos grupos que investigan la enseñanza de las ciencias dentro de un marco universitario. Por esta razon nos parece un tema interesante y también porque refleja en forma viva los vaivenes de la inserción teórica, de la metodología y de la formulación de una temática de investigación, pero siempre referidas a un problema surgido de la realidad de la saía de aula: ¿por qué es tan alto el porcentaje de alumnos repetidores en este curso?

Pretendemos mostrar tres puntos: a) cómo al adoptar ciertas teorías, el problema de investigación se transforma en diferentes formulaciones más o menos incluidas en los problemas que cobran sentido dentro de esta teoría; b) cómo las reflexiones son más o menos facilitadas o dificultadas en la medida en que los cuestionamientos pueden amenazar o no al núcleo de la teoría; y c) como ciertas limitaciones y datos de la realidad pueden llevar a mudanzas metodológicas y teóricas no previstas al comienzo.
Localicemos primero a nuestro pequeño grupo que trabaja en Brasil hace unos 10 años, con las dificultades de material, bibliografía y formación de alumnos, bastante típica del tercer mundo, o al menos de Latinoamérica.

Nuestro entusiasmo inicial por la teoría de Piaget nos permitio sacar el provecho que significa estar dentro de un paradigma teórico con una base amplia y sólida, como consideramos que es Ia Psicología y Epistemología Genética. Esto nos dio la posibilidad de poder formular preguntas de trabajo (como por ejemplo la primera, sobre el nivel de las operaciones lógicas de nuestros alumnos), con un método (la entrevista clínica o crítica utilizada por la escuela de Ginebra). No éramos conscientes aún de las limitaciones que este mismo hecho de estar dentro de un paradigma nos imponía: pero dejaremos estos comentarios para el final.

Provistos de estas herramientas realizamos un trabajo preliminar (Dibar Ure 1982) usando entrevistas clínicas variadas de temas que se prestaban bastante natiralmente a ser aplicados a estudiantes de ciencias. Los temas trabajados fueron: cuantificación de las probabi- 
lidades (Piaget 1951), péndulo y balanza y, posteriormente, proyección de sombras (Inhelder y Piaget 1955). Presentamos un esquema de éste y otros trabajos de esta línea que le siguieron, siempre referidos al aprendizaje de Mecánica Básica.

Los resultados del primer trabajo mostraban porcentajes que pudimos comparar razonablemente bien con los obtenidos en otros países. Solamente de 30 a $60 \%$ de nuestros alumnos parecían tener un razonamiento hipotético deductivo, característico de las operaciones formales. El resto presentaba razonamientos que po" dían ser asociados con operaciones concretas, o even. tualmente razonamientos intermediarios entre ambas. Notamos también que el desempeño variaba frente a diferentes pruebas.

Nos propusimos aplicar estos resultados preliminares a un curso de Mecánica Básica para Físicos (Queiroz y Dibar Ure 1981), dando mucho enfasis al rol activo del alumno en el proceso de enseñanza/aprendizaje. Esta tentativa se basaba en un buen número de laboratorios más abiertos, de listas de problemas que comenzaban sondeando hasta donde el alumno había llegado en su construcción de los conceptos y de la lógica, de trabajos grupales y pocas clases expositivas. Buscábamos enfatizar contradicciones que llevasen a cambios conceptuales y avances en el razonamiento.

Nuestra preocupación con la utilización de los resultados en el aula nos había llevado también a intentar desarrollar una versión escrita de las pruebas operatorias en la forma de un cuestionario para ser aplicado a grupos grandes en un tiempo reducido. De hecho si había algún nivel en que esto tenf́a sentido era justamente con un grupo como éste, altamente escolarizado $y$ en transicion hacia un nivel que puede independizarse del manipuleo indispensable para los niños en el período de las operaciones concretas.

Elegimos problemas en los cuales las justificaciones dadas por los alumnos replicasen los tipos de razonamientos encontrados en las entrevistas clínicas realizadas por Piaget (Inhelder y Piaget 1955). También nos preocupamos de que la respuesta a estos problemas no precisase de una lectura experimental para mostrar una ley física (como los de balanza y sombras que usamos sólo en entrevistas clínicas con material de laboratorio).

Dando continuidad a este trabajo y utilizando un delineamiento experimental cuidadoso, aplicamos un cuestionario escrito y realizamos una entrevista clínica sobre proyección de sombras con un grupo de 80 alumnos, pero cuidando de hacerlo con la mitad de ellos (tomados al azar) en la secuencia entrevista clínicacuestionario, y con la otra mitad en la secuencia inversa (cuestionario-entrevista clínica). En el cuestionario usamos 4 problemas que habíamos elegido en la etapa preliminar (sobre probabilidades, flexibilidad de varillas, combinaciones químicas y sobre proporciones en un problema de alturas). En la entrevista clínica elegimos el problema de proyección de sombras por comprender tanto proporciones como trabajo hipotético- deductivo en un contenido diferente al del cuestionario.

Nos sorprendió el siguiente resultado que aparecía con todas las preguntas del cuestionario, pero más nítidamente con relación a la de probabilidades: cuando la entrevista clínica precede al cuestionario, aparece una correlación entre los resultados de ambos. En la situación inversa, esta correlación desaparece. Podríamos pensar que esto se debe a que la entrevista, con su interacción con el material, permite actualizar los esquemas de razonamiento; pero esto daría una media mayor en el cuestionario para el grupo entrevista clínica-cuestionario que para el otro, y esto no ocurre: la media se mantiene significativamente igual. Esto parece mostrar que con la entrevista clínica precediendo, algunos alumnos deben mejorar y otros empeorar. Lo que sorprende entonces es el efecto organizador de la entrevista clínica sobre el cuestionario. En el momento en que hicimos esta investigación, no pudimos encontrar ninguna hipótesis sobre la naturaleza de este factor, que afecta los resultados tan fuertemente.

En principio entonces, este cuestionario no puede substituir a la entrevista clínica (pues sería equivalente a la situación en que el cuestionario la precede y donde no se ve ninguna correlación).

Resulta interesante ver, en retrospectiva, la dificultad que enfrentamos al estar dentro del paradigma piagetiano. Al encontrar un resuitado no esperado, preferimos inicialmente aceptar que el cuestionario efectivamente no podía substituir la entrevista clínica y archivarlo. Ahora bien, la entrevista clínica es un método flexible y muy valioso, pero aceptar sin cuestionar este resultado significa no sólo no pensar en substituirla sino abandonar la posibilidad de usar los cuestionarios como valiosos contrachequeos con esta población.

Vale la pena mencionar que el cuestionario también había mostrado tener una correlación con las concepciones alternativas, identificadas en forma escrita, medidas por el problema del malabarista (Viennot 1979). Esto sugería entonces que parecía ser más fácil realizar la mudanza conceptual al dominio de la explicación newtoniana para quien tenía acceso a los razonamientos formales. (Este trabajo no fue publicado justamente debido a las dudas surgidas sobre la validez del cuestionario escrito).

También estudiamos con esta misma población, las construcciones de los alumnos que son reveladas cuando resuelven un problema de 2 resortes en serie (Fauconnet 1981, Dibar Ure 1984); los resultados muestran la dificultad en el uso de la descripción matemática y, en particular, el problema de los sujetos que parecen "perderse" en las fórmulas substituyendo sin criterio, en oposición a aquéllos que aun cuando equivocados usan una estrategia consistente.

Llegamos entonces a cuestionamos dos aspectos de nuestro trabajo inicial, uno teórico y otro metodológico. El primero, metodologico, surge también a través de nuestros trabajos con otras poblaciones, especialmente adultos analfabetos y estudiantes universitarios 
más avanzados, y nos lleva a cuestionar que la entrevista clínica individual sea siempre el método más adecuado. El segundo cuestionamiento, teórico, generó varios encuentros con investigadores de otras dos universidades de nuestra región. Nos habíamos propliesto discutir la teoría piagetiana, especialmente en lo que respecta a la construcción de las operaciones causales (Piaget y García 1971) y los resultados de las investigaciones en Nociones Alternativas provenientes de la Enseñanza de la Física. En particular discutimos en qué medida estas dos posiciones pueden ser consideradas complementarias, enriqueciéndose mutuamente, y en qué medida entran en contradicción.

De estos encuentros permanecieron en nuestro grupo ciertos temas en discusion y ciertos cuestionamientos:

a) ¿La descripción por ta construcción de las "operaciones causales" explica realmente las dificultades introducidas por la "respuesta del objeto físico"?

b) ¿Qué đecir sobre la persistencia de muchos "modelos alternativos"? ¿Estos modelos no pueden convivir con razonamientos correctos, como ocurrió en la Historia de la Física?

Se encuentra con insistencia, al repetir experiencias ya realizadas por Piaget, que los primeros estadios cognitivos son reencontrados; pero al llegar al último, encontramos mucho más comunes las respuestas tipo alternativas, en cuanto Piaget cita siempre ejemplos que parecen sugerir que los alumnos llegan a los conceptos usados por las ciencias.

c) El "sujeto epistémico", descrito por Piaget, puede ser muy útil como una primera aproximación, pero debe ser enriquecido en su funcionamiento si hemos de describir el razonamiento del alumno en el proceso de aprender ciencias. En otro trabajo (Dibar Ure y Castorina 1989) discutimos un estudio de M. Carretero (1984) sobre flotación de cuerpos. Vemos que la influencia de los contenidos de las hipótesis sobre los procedimientos de prueba - cuya evolución está asociada a las operaciones lógico-matemáticas - no es pequeña sino que es por lo menos una variable tan relevante como la secuencia de operaciones. Esto nos acerca quizás a la tesis de L. Viennot acerca de la persistencia de las nociones espontáneas aunque puedan modificarse los métodos del pensamiento experimental a lo largo dei desarrollo.

d) En su libro Las explicaciones causales (1971), Piaget y García se preocupan principalmente en mostrar que las operaciones causales, que describen la construcción del conocimiento acerca del mundo físico, se construyen junto con las operaciones lógicas que corresponden a la elaboración del razonamiento del sujeto, sin primacía de unas ni de otras. Esta demostración nos parece sujeta a definiciones pobremente discutidas usando los datos experimentales (por ejemplo causalidad, legalidad, atribución, aplicación y necesidad).

Cuestionamos especialmente si la necesidad, que Piaget asocia a la causalidad y no a la legalidad, no sería el centro de esta discusión; en efecto, la causalidad acrecentada de esta necesidad lógica se integra armónicamente al modelo principal, que exige el desarrollo paralelo de los dos tipos de operación sin primacía de una ni de la otra, pero deja de describir la multiplicidad de modelos físicos lógicamente posibles.

No podemos entrar aquí, en este breve resumen, a desarrollar los puntos de acuerdo y los que permanecieron en discusión. Prevaleció sí, una búsqueda, una posibilidad de enriquecimiento de dichos temas, una posibilidad de tomar ciertos puntos de la teoría piagetiana con respecto al conocimiento físico como una primera aproximación, que debe ser enriquecida y cuestionada para describir los razonamientos y las construcciones de los alumnos.

A partir de este momento, surgío entonces en nuestro grupo la idea de retomar las experiencias con alumnos de la primera física, con una visión más amplia. Retomamos nuestra pregunta inicial sobre el porqué de la repetición.

Cuando cambiamos al curso teniendo en cuenta las dificultades en el razonamiento y en realizar la mudanza conceptual, el índice de reprobación cambió poco. Nos preguntamos entonces que otras variables son importantes. ¿Cómo podemos saber más sobre la posición del alumno frente al conocimiento? ¿Y los profesores? $\measuredangle$ Cómo ven ellos este problema? Algunos resultados preliminares de este trabajo que nuestro grupo está realizando fueron presentados en el III Simposio de Enseñanza de las Ciencias y de las Matemáticas (Santiago de Compostela, 1989), en sesión de paneles.

Vale la pena aquí mencionar uno de ellos: se puede ver, a traves de entrevistas y de discusiones grupales que muchos alumnos, en particular los repetidores, piden no entrar en contradicciones ni sentirse perdidos, ser protegidos, y que se les dé el conocimiento. Pero esto está justamente en oposicion con lo que buscan los profesores que están al tanto de las últimas investigaciones, pues hay un acuerdo casi total en que el alumno debe construir el conocimiento y que hay que promover estas contradicciones o conflictos para hacer avanzar los cambios conceptuales y, con ello, la comprensión y la capacidad de razonar en física de los alumnos.

Finalmente, en esta búsqueda de las causas, que influyen en la posición del alumno frente al curso, vimos que aparece fuertemente la relacion profesor-alumno. En el contexto de esta discusión, surgió entonces la idea de volver a pensar en la correlación entre el cuestionario y la entrevista clínica: ino será que la entrevista clínica, como interacción entre dos personas, modifica el razonamiento en un cuestionario posterior, que el alumno sabe que proviene del mismo equipo? Esta influencia no sería siempre para mejor, ni siempre para peor, sino que saca al alumno de una respuesta escolar (que es el código del cuestionario), haciendo aparecer aquel tipo de respuesta que fue elaborada en interacción con el entrevistador y con material de laboratorio. Una entrevista demorada, que usa material de laboratorio, y hecha por un entrevista- 
dor dedicado que da atención, valora un tipo de razonamiento que el alumno repite días más tarde en el cuestionario, en otro contenido. Podemos imaginar también tal vez lo opuesto, alumnos a los que no les gusta ser observados y trabajan más a gusto cuando están solos; al hacer la entrevista clínica antes del cuestionario, no conseguirían en éste un desarrollo tan bueno como cuando no se han sentido observados. En definitiva, pueden aparecer otros factores, como la interacción o la relación con otra persona y no sólo con el conocimiento. Por otro lado, nos podemos preguntar si noes a esto mismo a lo que se refieren los piagetianos cuando se habla de una "enirevista clínica bien hecha", con un buen "rapport" con el estudiante y si no son las mismas variables que cuando los profesores hablan de la importancia de una posición "positiva" del profesor con respecto a la clase, y dicen que es importante que un profesor "crea en lo que está haciendo".

Resumiendo, esta visión retrospectiva del trabajo con universitarios del primer año fue de alguna manera difícil; pero nos mostró que era necesaria una apertura en cuanto a nuestra visión inicial más piagetiana, no

\section{REFERENCIAS BIBLIOGRÁFICAS}

CARRETERO, M., 1984. De la larga distancia que separa la suposición de la certeza, en lecturas de Psicología del Pensamiento. Razonamiento. Solución de Problemas y Desarrollo Cognitivo, Compilación de M. Carretero y J. A. García Madruga. (Alianza Editorial: Madrid), pp. 32 I339.

DIBAR URE, M.C., 1982. Estudio Preliminar do Nível Operatorio de Estudantes Universitários, Rev. Brasileira de Fisica, I2(2), pp. 363-374.

DIBAR URE, M.C., 1984. Brazilian students' answer to a problem in Mechanics, Procedings GIREP 84, pp. 234239.

DIBAR URE, M.C., y CASTORINA, J. A., 1989. Algunas reflexiones sobre la relación entre hipótesis y procedimientos en la Física Infantil, Presentación deI Ill Congreso Internacional sobre Enseñanza de las ciencias, Santiago de Compostela, 1989.

DIBAR URE, M.C. y QUEIROZ, G.R.P.C., 1984. Estudio del nivel operatorio de Estudiantes Universitarios, Enseñanza de la Ciencias, 2 (3), pp. 187-193.

FAUCONNET, S., 1981. Etude de Resolution de Problèmes: Quelques problèmes de même structure en Physique, Thesis, Universite Paris VII. sólo para incluir la importancia del fenómeno físico y sus modelos, sino también para otros factores no estrictamente relacionados con el razonamiento, como la interacción profesor-alumno o entrevistador-alumno.

A este respecto conviene recordar un párrafo de $\mathrm{L}$. Viennot (1989) en su artículo "La Didáctica en Ia Enseñanza Superior. ¿Para qué?". Al hablar sobre "los principios convenidos entre casi la totalidad de quienes... han elegido la enseñanza como objeto de investigación... se trata de estudiar aspectos de la enseñanza de la física relacionados con el contenido de esta asignatura" y, "por esta razón, quedan relativamente excluidos del campo de la investigación determinados aspectos susceptibles de ser pertinentes, tales como la motivación, el contexto sociopsicológico, etc.".

Nuestros datos nos sugieren que tal separación del campo de estudio no parece ser posible. Sin tener en cuenta otros factores que podemos relacionar con los que L. Viennot llama "motivacionales" o "sociopsicológicos" algunos de nuestros resultados no pueden ser explicados.
INHELDER, B. y PIAGET, J., 1955. De la logique de l'en: fant à la logique de l'adolescent. (PUF: Paris). (Traduc. ed. Paidos), cap. 5.

PIAGET, J., 1926. La représentation du monde chez l'enfant (Alcan: Paris). (Introduction. Problèmes et méthode).

PIAGET, J., 1951. La Genèse de l'ideée de Hasard chez l'enfant. (PUF: Paris).

PIAGET, J. y GARCÍA, R., 1971. Las Explicaciones Causales. (Barral Editora, Siglo XXI: Barcelona).

QUEIROZ, G.R.P. y DIBAR URE, M.C., 1981. Uma experiencia de ensino na primeira cadeira de Física Básica na Universidade, Rev. de sino de Física, 3(4), pp. 3-11.

VIENNOT, L., 1979. Spontaneous reasoning in elementary dynamics, European Journal Science Education, 1(2), pp. 205-222.

VIHN-BANG, 1966. La Méthode Clinique et la recherche en psychologie de l'enfant, en Psycologie et épistémologie génétiques, (ouvrage collectif). (Dunod: Paris).

VIENNOT, L., 1989. La didáctica en la Enseñanza Superior. ¿Para qué?, Enseñanza de las Ciencias, 7(1). 